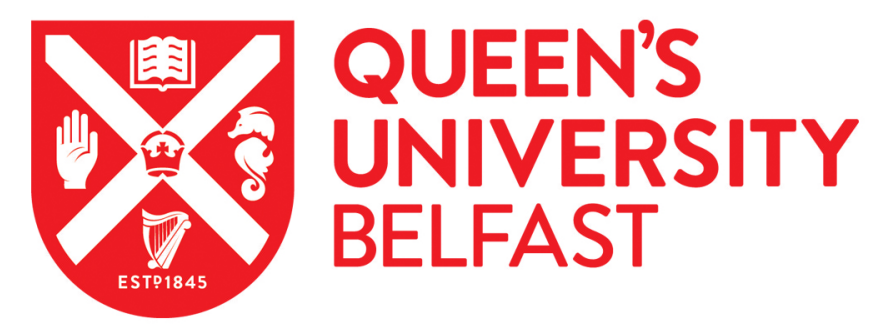

\title{
Effect of quantization of vibrations on the structural properties of crystals
}

Scivetti, I., Gidopoulos, N., \& Kohanoff, J. (2008). Effect of quantization of vibrations on the structural properties of crystals. Physical Review B (Condensed Matter), 78(22), [224108].

https://doi.org/10.1103/PhysRevB.78.224108

Published in:

Physical Review B (Condensed Matter)

Document Version:

Publisher's PDF, also known as Version of record

Queen's University Belfast - Research Portal:

Link to publication record in Queen's University Belfast Research Portal

Publisher rights

(2008 The American Physical Society

\section{General rights}

Copyright for the publications made accessible via the Queen's University Belfast Research Portal is retained by the author(s) and / or other copyright owners and it is a condition of accessing these publications that users recognise and abide by the legal requirements associated with these rights.

Take down policy

The Research Portal is Queen's institutional repository that provides access to Queen's research output. Every effort has been made to ensure that content in the Research Portal does not infringe any person's rights, or applicable UK laws. If you discover content in the Research Portal that you believe breaches copyright or violates any law, please contact openaccess@qub.ac.uk. 


\title{
Effect of quantization of vibrations on the structural properties of crystals
}

\author{
Iván Scivetti, ${ }^{1}$ Nikitas Gidopoulos, ${ }^{2}$ and Jorge Kohanoff ${ }^{1, *}$ \\ ${ }^{1}$ Atomistic Simulation Centre, Queen's University Belfast, Belfast BT7 1NN, Northern Ireland, United Kingdom \\ ${ }^{2} I S I S$ Facility, Rutherford Appleton Laboratory, Chilton, Didcot, Oxon OX11 OQX, England, United Kingdom
}

(Received 2 October 2008; published 29 December 2008)

\begin{abstract}
We study the structural effects produced by the quantization of vibrational degrees of freedom in periodic crystals at zero temperature. To this end we introduce a methodology based on mapping a suitable subspace of the vibrational manifold and solving the Schrödinger equation in it. A number of increasingly accurate approximations ranging from the quasiharmonic approximation (QHA) to the vibrational self-consistent field (VSCF) method and the exact solution are described. A thorough analysis of the approximations is presented for model monatomic and hydrogen-bonded chains, and results are presented for a linear H-F chain where the potential-energy surface is obtained via first-principles electronic structure calculations. We focus on quantum nuclear effects on the lattice constant and show that the VSCF is an excellent approximation, meaning that correlation between modes is not extremely important. The QHA is excellent for covalently bonded mildly anharmonic systems, but it fails for hydrogen-bonded ones. In the latter, the zero-point energy exhibits a nonanalytic behavior at the lattice constant where the $\mathrm{H}$ atoms center, which leads to a spurious secondary minimum in the quantum-corrected energy curve. An inexpensive anharmonic approximation of noninteracting modes appears to produce rather good results for hydrogen-bonded chains for small system sizes. However, it converges to the incorrect QHA results for increasing size. Isotope effects are studied for the first-principles H-F chain. We show how the lattice constant and the H-F distance increase with decreasing mass and how the QHA proves to be insufficient to reproduce this behavior.
\end{abstract}

DOI: $10.1103 /$ PhysRevB.78.224108

PACS number(s): 61.50.Ah

\section{INTRODUCTION}

Structural properties of solids such as interatomic distances, bond angles, and equilibrium lattice parameters are customarily calculated by assuming that atomic nuclei (or ionic cores) behave as classical particles. Within this framework, the electronic problem is solved for a fixed configuration of clamped nuclei. The resulting ground-state electronic density allows for the computation of the forces acting on the nuclei through the Hellman-Feynman theorem. These forces are then used to determine the equilibrium configuration. While this is justified for a large variety of systems of interest, the assumption of classical nuclei becomes questionable whenever light atoms such as hydrogen are involved. Moreover, this approximation cannot address isotope effects; the ground-state electronic energy does not depend on the nuclear masses but only on the atomic numbers. A widely used approach to introduce the quantum nature of the nuclei is the quasiharmonic approximation (QHA) ${ }^{1-3}$ In this approximation, the nuclear ground-state energy is supplemented with the zero-point energy (ZPE) corresponding to harmonic nuclear vibrations. This is based on a second-order Taylor expansion of the potential-energy surface (PES) in terms of the atomic coordinates around the equilibrium configuration of the nuclei. The resulting dynamical matrix, given by the second derivatives of the potential with respect to the nuclear coordinates, can be diagonalized thus leading to a set of orthogonal eigenvectors or normal modes $\zeta_{l}$, together with the corresponding set of frequencies $\omega_{l}{ }^{4}$

To simulate an infinite crystal, it is customary to apply periodic boundary conditions (PBCs) on the unit cell. ${ }^{4}$ Therefore, a crystal described by a basis of $N$ atoms in the unit cell is characterized by $3 N$ vibrational bands $\omega_{l}(\mathbf{k})$, where $\mathbf{k}$ is a wave vector in the phonon Brillouin zone (BZ). Within this framework, the QHA energy at zero temperature, $E^{\mathrm{QHA}}$, is defined as

$$
E^{\mathrm{QHA}}(V)=E^{\mathrm{cl}}(V)+\frac{\hbar}{2} \sum_{l=1}^{3 N} \int_{\mathrm{BZ}} g(\mathbf{k}) \omega_{l}(\mathbf{k}, V) d \mathbf{k},
$$

where $V$ is the volume of the system, $g(\mathbf{k})$ is the density of states, and the integral extends to the Brillouin zone. The first term in the right-hand side (RHS) is the ground-state energy for the equilibrium configuration at volume $V$. The second term includes the quantum nature of the nuclei through the harmonic ZPE, which also depends on $V$ in a way that is characteristic of the type of bonding. For covalent bonding it increases upon compression, while for hydrogen bonding there is a competition between increasing and decreasing frequencies. The combination of ground-state and zero-point energies leads to a modified $E^{\mathrm{QHA}}$ vs $V$ curve, in contrast to $E^{\mathrm{cl}}$ vs $V$, with a minimum located at a volume corrected by quantum nuclear effects. The QHA has the additional advantage of lending itself naturally to the simultaneous incorporation of quantum and thermal effects. ${ }^{5}$

In the harmonic approximation the ground-state nuclear wave function is a product of single-mode Gaussians on the vibrational normal-mode variables $\zeta_{l}$, and the expectation value of the nuclear coordinates coincides exactly with the classical nuclear configuration. Apart from variations mediated by volume changes, internal structural parameters (distances and angles) are unaffected in the QHA. It is then expected that the QHA breaks down when treating highly anharmonic systems.

Additional terms in the Taylor expansion lead to anharmonicity, which can come essentially in two forms. The simplest one is intramode anharmonicity when the vibrational modes remain noninteracting but the potential felt by one or more modes cannot be approximated by a quadratic expression. This type can be included in a rather simple way. Nor- 
mal modes can be used as coordinates to map the PES beyond the harmonic level and the energy of each mode obtained by solving a set of independent one-dimensional (1D) Schrödinger equations. The second type of anharmonicity is due to mode coupling. When the excursions along a vibrational normal coordinate are large, the approximation of small oscillations implicit in the second-order expansion breaks down. Successive terms in the expansion involve products of modes of the form $\zeta_{l}^{m} \zeta_{l^{\prime}}^{n}$ as well as higher-order terms involving more than two modes. Which groups of modes are more strongly coupled depends very much on the specific system. In general there are no rules to find out $a$ priori which couplings need to be considered. A possible strategy is to displace the system along one mode, say $\zeta_{1}$, by an amount $\epsilon$, and then optimize the atomic coordinates under the constraint $\zeta_{1}=\epsilon$. By projecting the displacements obtained for the optimized configuration onto the original normal modes, one can identify and select the modes with the largest projection. ${ }^{6}$ The main goal of this paper is to evaluate the quality of the QHA and, where required, to provide improved schemes at an affordable computational cost. To this end, we will include anharmonicity by solving the vibrational Schrödinger equation in a sequence of increasingly accurate approximations and compare to the QHA results.

An additional issue that arises when computing the ZPE is that the BZ integrals in Eq. (1) must be replaced with appropriate averages over a finite set of representative points in the BZ. This can be done in several ways. The most accurate one is to compute the force constants in real space and then use them to obtain the dynamical matrix at an arbitrarily dense mesh of $\mathbf{k}$ points in the BZ. This requires calculations in large supercells or, alternatively, linear-response calculations. ${ }^{7}$ On the other extreme, the crudest approximation is to include only the BZ-center modes of the unit cell, while improvements can be achieved by considering BZcenter modes of larger supercells. The advantage of the latter is that it is easier to extend to anharmonic situations. The second goal of this paper is to analyze how large a supercell should be in order to reproduce the equilibrium structure obtained with converged BZ averaging. This issue has rarely been discussed in the literature.

In order to answer these questions we have studied two qualitatively different models: a one-dimensional monatomic chain and a diatomic linear chain that models a double-well hydrogen-bonded system. In Sec. III A we show that, as usually done in the literature, systems characterized by covalent bonding can be safely described within the quasiharmonic approximation using a rather coarse BZ sampling. In Sec. III $\mathrm{B}$, in contrast, we show that hydrogen-bonded systems require some level of anharmonicity in their treatment. An anomaly in the ZPE appears when the protons center in the $\mathrm{H}$ bonds upon compression. This produces an unusual behavior of the QHA, which can lead to a secondary spurious minimum in the energy-volume curve. We then study a realistic linear F-H $\cdots$ F chain where the PES has been obtained from first-principles calculations (Sec. III C). We study quantum effects, in particular the isotope effect on equilibrium lattice constant and internal geometry (H-F distance) occurring when the nuclear masses are modified (Sec. III E). In this case, an inexpensive anharmonic approximation that neglects mode coupling appears to produce an accurate lattice constant. In Sec. IV we present our conclusions and elaborate on possible extensions. In the following, we introduce the theoretical and computational approaches used in the present work.

\section{METHODS AND APPROXIMATIONS}

In this paper we will only consider one-dimensional systems for the sake of simplicity. Although computationally more demanding, extensions to higher dimensions are straightforward. In the QHA, the energy for a onedimensional system containing $N$ particles is

$$
E^{\mathrm{QHA}}(a)=E^{\mathrm{cl}}(a)+\frac{\hbar}{2} \sum_{i=1}^{N-1} \omega_{i}(a),
$$

where $a$ is the lattice constant, which plays the role of the volume, and $E^{\mathrm{cl}}(a)$ is the ground-state electronic energy in the approximation of classical nuclei (classical energy). The vibrational frequencies $\omega_{i}(a)$ are obtained by assuming PBCs, i.e., we consider a cyclic chain. The sum runs up to $N-1$ because the vibrational mode corresponding to the rigid translation of the whole chain has zero frequency.

In the harmonic approximation there is no need to consider explicitly the $N$-atom chain. The same result can be obtained by studying monatomic or diatomic cells subjected to PBC. This equivalence is explained thoroughly in most solid-state books. ${ }^{4}$ Here we just quote the main result, i.e., the dispersion relations that express the frequency as a function of wave number $k$ in the one-dimensional phonon Brillouin zone. For a monatomic chain the dispersion relation is

$$
\omega(k, a)=2 \sqrt{\frac{V^{\prime \prime}(a)}{m}}\left|\sin \left(\frac{k a}{2}\right)\right|
$$

where $m$ is the mass of the particles, $k=\pi n /(N-1) a$, and $n$ $=0,1, \ldots, N-1$. The quantity $V^{\prime \prime}(a)$ is the second derivative (curvature) of the interaction potential $V$ evaluated at the equilibrium configuration and depends on the lattice constant $a$. The frequencies obtained in this way correspond exactly with those of an $N$-atom cyclic chain. In the limit of $N \rightarrow \infty$, the QHA energy for a monatomic chain is

$$
E^{\mathrm{QHA}}(a)=E^{\mathrm{cl}}(a)+\frac{\hbar}{2} \int_{\mathrm{BZ}} g(k) \omega(k, a) d k .
$$

The case of a diatomic linear chain is analogous, with the difference that there are two dispersion relations, representing the acoustic and optical branches. The expressions are still analytic but slightly more complicated. ${ }^{4}$

For a hydrogen-bonded diatomic chain such as F-H $\cdots \mathrm{F}$ the harmonic approximation can only be carried out at one of the two equivalent global minima. If the barrier is so high that tunneling is unlikely, then this is a reasonable approximation. However, if tunneling is important, anharmonic effects start to play a significant role and the QHA breaks down. By symmetry, one could also choose a reference configuration at the top of the inversion barrier, i.e., with the $\mathrm{H}$ atoms centered between $\mathrm{F}$ atoms. This configuration is a sta- 
tionary point of the PES, but the QHA breaks down because a whole portion of the optical phonon branch is unstable around zone center.

The calculated normal modes can now be used to map the true PES beyond the harmonic approximation. In general, this is a complicated mutidimensional fitting problem that requires specific techniques such as the product representation. ${ }^{8} \mathrm{~A}$ much simpler alternative is to keep considering the normal coordinates as noninteracting but anharmonic. This corresponds to pure intramode anharmonicity. The full-dimensional vibrational problem is then reduced to mapping the one-dimensional potential for every normal coordinate keeping the other normal coordinates at their classical value, i.e., zero. Then, the 1D Schrödinger equation is solved for each one of these potentials and their ground-state energies are added up. We call this the anharmonic approximation (ANHA).

At the other end of the theoretical spectrum, the problem of interacting phonons can be tackled exactly by solving the $(N-1)$-dimensional Schrödinger equation. This task can be accomplished with little effort for 3 degrees of freedom but finds a hard wall at six. This means that, in the onedimensional case, one can routinely compute the exact solution for a four-atom supercell but cannot go beyond sevenatom chains if a significant fraction of the eigenvalue spectrum is required. Stochastic methods such as diffusion Monte Carlo can, however, be used to compute the lowenergy region of the spectrum, as it has been done for vibrations in molecular systems. ${ }^{9}$

Here we have chosen the strategy of solving the vibrational Schrödinger equation using a discrete variable representation. We used Lagrange grids based on a combination of Cartesian and Hermite orthogonal polynomials ${ }^{10,11}$ for double-well and single-well potentials, respectively. When solving the Schrödinger equation exactly, a maximum of 3 vibrational degrees of freedom was considered. This implies that the largest monatomic linear chain we studied was made of four unit cells, while the largest diatomic chain contained two unit cells.

We have also studied a class of approximations to the full-dimensional solution inspired by the vibrational selfconsistent field (VSCF) method, ${ }^{12}$ which is frequently used to compute vibrational excitation spectra of large molecules. ${ }^{13}$ In the VSCF method the normal coordinates are taken as completely uncorrelated and the total wave function is written as a product of single-mode wave functions,

$$
\Psi\left(\zeta_{1}, \ldots, \zeta_{N-1}\right)=\prod_{i=1}^{N-1} \phi_{i}\left(\zeta_{i}\right)
$$

in the same spirit of the Hartree approximation to the problem of interacting electrons. Contrary to the harmonic and anharmonic approximations, each mode now feels the presence of the other modes but in a mean-field way. The VSCF equations are

$$
\left[-\frac{\hbar^{2}}{2 M} \frac{d^{2}}{d \zeta_{i}^{2}}+V_{i}\left(\zeta_{i}\right)\right] \phi_{i}\left(\zeta_{i}\right)=\epsilon_{i} \phi_{i}\left(\zeta_{i}\right),
$$

where

$$
V_{i}\left(\zeta_{i}\right)=\int \cdots \int V\left(\zeta_{1}, \ldots, \zeta_{N-1}\right) \prod_{j=1, j \neq i}^{N-1}\left|\phi_{j}\left(\zeta_{j}\right)\right|^{2} d \zeta_{j}
$$

are the mean-field potentials for each mode. For a 1D system the energy in the VSCF method is given by

$$
E_{\mathrm{VSCF}}=\sum_{i=1}^{N-1} \epsilon_{i}-(N-2) \int V_{i}(\zeta)\left|\phi_{i}(\zeta)\right|^{2} d \zeta,
$$

where the second term corrects for double counting in the sum of eigenvalues. Notice that the integral in this term should be independent of $i$, within numerical accuracy.

The determination of the mean-field potential requires the computation of $(N-2)$-dimensional integrals, which rapidly becomes an expensive operation. Therefore, it is desirable to find either simpler approximations or some way of evaluating these integrals at a reduced computational cost. The ANHA mentioned above removes this limitation by replacing the single-mode densities $\left|\phi_{j}\left(\zeta_{j}\right)\right|^{2}$ in Eq. (7) with delta functions centered at $\zeta_{j}=0$. With this, the ANHA can be carried onto much larger systems.

The quality of the VSCF results depends on the choice of coordinates. If the system is only weakly anharmonic, then the classical normal modes are a very reasonable choice. This is not necessarily the case when anharmonicity becomes important. The question arises on whether it is still possible to find a set of orthogonal coordinates that are only weakly correlated, or whether correlation is intrinsic to the problem and cannot be significantly reduced by a clever choice of coordinates. Within the VSCF context, finding the optimally decorrelated coordinates is equivalent to requiring that these minimize $E_{\mathrm{VSCF}}$. The optimal coordinates $\left\{\zeta_{i}\right\}$ can be obtained by solving the VSCF problem for a linear combination of normal modes $\zeta_{i}^{\prime}=\sum_{j} Z_{i j} \zeta_{j}$ and minimizing $E_{\mathrm{VSCF}}$ with respect to the coefficients $Z_{i j}$ of the rotation matrix, subjected to orthonormality constraints. ${ }^{14}$ This task can be accomplished by constrained multidimensional optimization algorithms. ${ }^{15}$ Nevertheless, there is no clear evidence of a general method for obtaining the optimal set of coordinates. In this work we analyze the quality of the VSCF approximation with respect to the choice of vibrational coordinates for the $a b$ initio hydrogen-bonded linear chain.

\section{RESULTS}

\section{A. Model monatomic chain}

We first considered a periodic monatomic chain where the atoms interact via a Morse potential of the form

$$
V(x)=D\left(1-e^{-b\left(x-a_{0}\right)}\right)^{2},
$$

where parameter $D$ represents the binding energy of a dimer, $a_{0}$ is the location of the minimum of the potential, which corresponds to the classical lattice constant, and $b$ is a parameter that determines the curvature of the potential through the relation $V^{\prime \prime}\left(a_{0}\right)=2 D b^{2}$. The Morse potential is intrinsically anharmonic and allows us to study the effect of quantization of the vibrations on the lattice constant. For the various approximations described in Sec. II we calculated the 


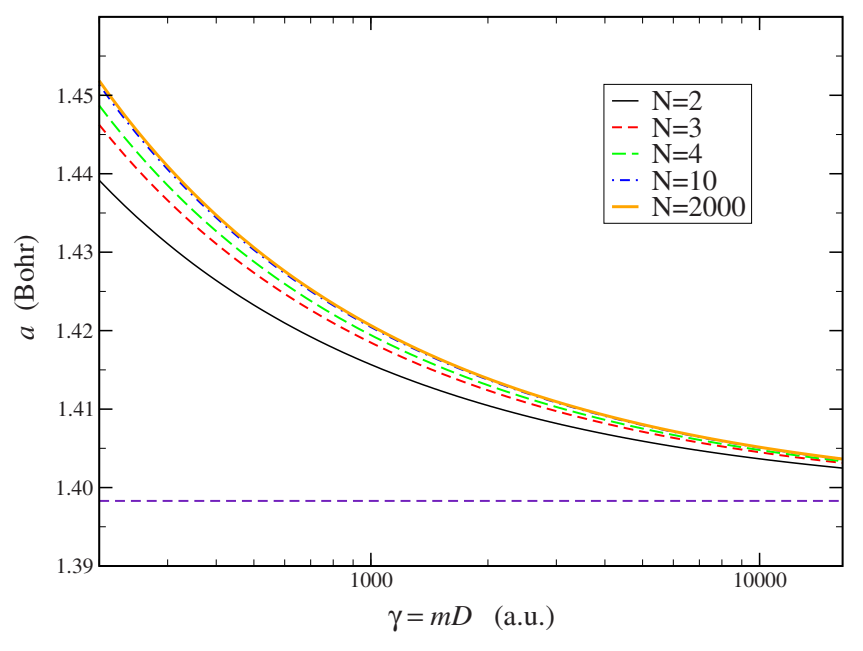

FIG. 1. (Color online) QHA lattice constant as a function of $\gamma$, for increasing number of atoms in the supercell: $N=2$ (black thin solid line), $N=3$ (red short-dashed line), $N=4$ (green long-dashed line), $N=10$ (blue dotted-dashed line), and $N=2000$ (orange thick solid-light gray line). The dashed horizontal line represents the classical value.

quantum-corrected energy as a function of the lattice parameter $a$ and then determined the minimum of the $E$ vs $a$ curve.

In the solution of Schrödinger equation, the extent of quantum nuclear effects can be measured by the product $\gamma$ $=m D$, where $m$ is the mass of the particles and $D$ is the energy scale of the potential. Small values of $\gamma$ lead to important quantum effects, becoming less relevant as $\gamma$ increases and eventually converging toward the classical results for $\gamma \rightarrow \infty$. Therefore, we report our results in terms of this quantity. In this work we have used $a_{0}=1.3983$ bohr and $b=2.9864$ bohr $^{-1}$.

We first analyzed the effect of Brillouin-zone sampling on the equilibrium lattice constant. For the QHA this can be done for an arbitrarily large number $N$ of $k$ points in the BZ, which is equivalent to considering a supercell containing an equal number $N$ of unit cells. In Fig. 1 we show the convergence pattern of the QHA lattice constant as a function of $\gamma$ for increasing values of $N$.

It is obvious that quantum effects are more important for smaller masses and for weaker interaction potentials. For $\mathrm{H}$ atoms and $D=2 \mathrm{eV}$ ( $\gamma=135$ a.u.) we are in the region to the left of Fig. 1, where the quantum-corrected lattice constant is about $4 \%$ larger than the classical value (horizontal line). It can be seen that, for this type of potential, ten cells are already sufficient to reproduce the infinite crystal lattice constant to high accuracy. However, four cells already produced excellent results and two cells underestimated $a$ in less than $1 \%$.

In order to illustrate the origin of the lattice expansion, we show in Fig. 2 the various energy curves for a four-atom supercell as a function of the lattice parameter for $\gamma$ $=304.97$ a.u. The classical energy curve $E^{\mathrm{cl}}(a)$ (black solid line) exhibits a minimum at the equilibrium lattice constant, where the one-dimensional pressure $P=-d E^{\mathrm{cl}}(a) / d a$ vanishes. The ZPE (red dotted-dashed line) depends on the lattice constant through the curvature of the potential. Upon

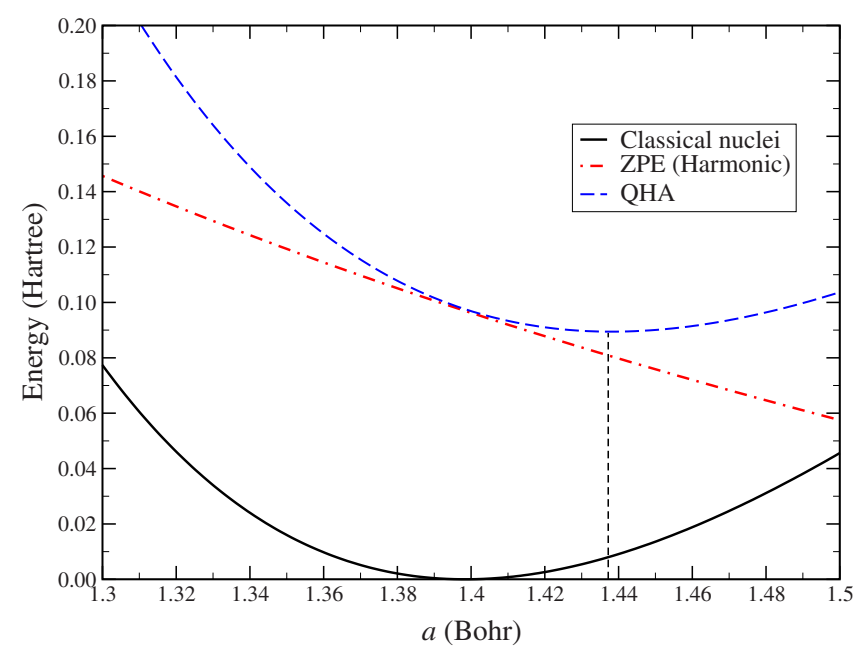

FIG. 2. (Color online) Contribution of the harmonic ZPE as a function the lattice parameter for a four-atom supercell and $\gamma$ $=304.97$ a.u. Since the ZPE increases as $a$ decreases (red dotteddashed line), the QHA lattice parameter (vertical dashed line) corresponding to the minimum of the QHA energy curve (blue dashed line) is expanded.

compression (reducing $a$ ), the chain becomes stiffer, all frequencies increase, and so does the ZPE. Therefore, the QHA energy given by the sum of the two terms in Eq. (4), and represented by the blue dashed curve, exhibits a minimum at an expanded lattice constant. This effect is similar to thermal expansion, but the origin is purely quantum mechanical. It can also be ascribed to a zero-point pressure originated in the dependence of the ZPE on the lattice constant.

To evaluate the quality of the QHA we calculated the equilibrium lattice constant in the ANHA and VSCF approximations and exactly for a four-atom supercell. The differences between these results and the QHA lattice parameter $\Delta a$ are reported in Fig. 3. It can be seen that these differences are 1 order of magnitude smaller than those arising from a

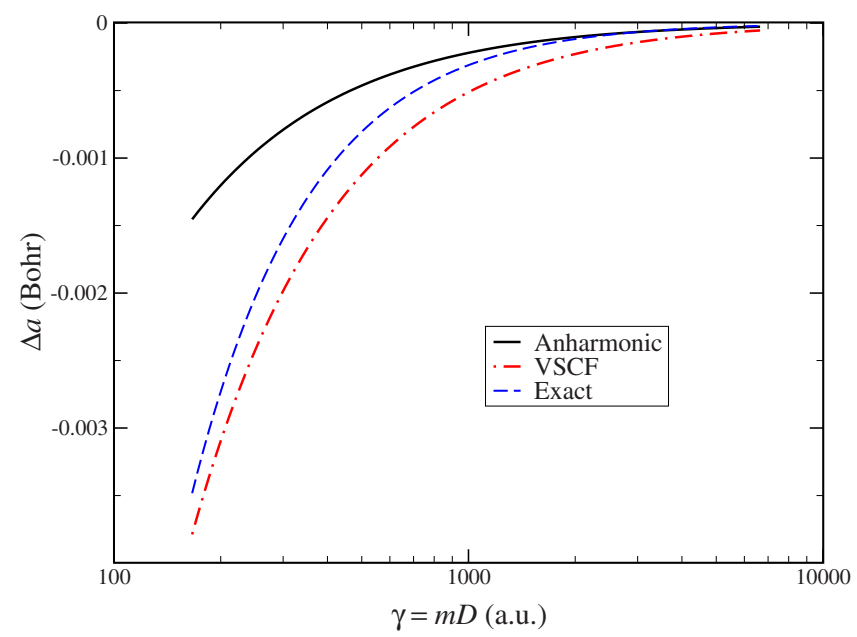

FIG. 3. (Color online) Difference in equilibrium lattice constant with respect to the QHA value, as a function $\gamma$ in a four-atom supercell. The black solid curve is for the ANHA, the red (dotteddashed) line is for the VSCF, and the blue (long-dashed) line is for the exact calculation. 
poor sampling of the BZ. These observations justify the approach commonly used to determine the quantum-corrected lattice constant of solids, i.e., that of computing the harmonic ZPE for a coarse BZ sampling, preferably only the $\Gamma$ point, adding it to the electronic ground-state energy corresponding to the equilibrium configuration and obtaining the lattice constant as the minimum of the sum. As we shall see now, however, this procedure is not necessarily useful for very anharmonic systems such as hydrogen-bonded crystals.

\section{B. Model hydrogen-bonded chain}

As an extension to highly anharmonic systems, we considered a model for a hydrogen-bonded diatomic chain where the light atoms are generally hydrogen and the heavy atoms can be oxygen or fluorine. For the sake of simplicity and to be consistent with Sec. III C, we call them $\mathrm{H}$ and F, respectively. For the F-H potential we retain a Morse type of interaction [Eq. (9)]. For sufficiently large F-F distances the $\mathrm{H}$ atoms feel attracted to one or the other $\mathrm{F}$ atom rather than being shared between them. Therefore, the $\mathrm{H}$ atoms in the chain are subjected to a double-well potential. This potential has an important property for what concerns isotope effects: the barrier between the two wells becomes lower when the two neighboring $\mathrm{F}$ atoms approach each other. This is an essential ingredient, which allows for an easier migration of the protons upon compression. The $\mathrm{F}$ displacements $\left(\rho_{i}\right)$ are measured from the given lattice parameter. On the other hand, the $\mathrm{H}$ displacements $\left(u_{i}\right)$ are measured from the midpoint between the equilibrium positions of the F atoms. Displacements $\rho_{i}$ and $u_{i}$ are continuous variables associated to discrete lattice sites $i$. With this, the double Morse contribution to the PES is

$$
\begin{aligned}
\mathcal{E}_{\mathrm{F}-\mathrm{H}}= & \sum_{i=1}^{N} D\left(1-e^{-b\left(a / 2+u_{i}-\rho_{i}-r_{e}\right)}\right)^{2} \\
& +\sum_{i=1}^{N-1} D\left(1-e^{-b\left(a / 2-u_{i}+\rho_{i+1}-r_{e}\right)}\right)^{2} \\
& +D\left(1-e^{-b\left(a / 2+u_{N}-\rho_{1}-r_{e}\right)}\right)^{2}
\end{aligned}
$$

where $N$ is the number of sites and $a$ is the lattice parameter. The potential parameters are the well depth $D$ and the position of the minima $r_{e}$ measured from the $\mathrm{F}$ atoms. The effects of PBCs are included in the last term on the RHS.

When the $\mathrm{H}$ atom sits off center it forms a neutral unit with the $\mathrm{F}$ atom and another $\mathrm{H}$ atom on the opposite side of the $\mathrm{F}$ is not welcome due to electronic closed-shell repulsion effects. A similar effect occurs in ice, where oxygens make two strong covalent bonds with $\mathrm{H}$ atoms to form the water molecules and two weaker hydrogen bonds with neighboring molecules. This, however, is not taken into account by the Morse potential. To introduce this feature, usually known as Pauling's "ice rules," it is necessary to include an interaction between the $\mathrm{H}$ atoms that discourages them from approaching the same $\mathrm{F}$ atom. There are several possible ways of doing this. A popular choice is to include a harmonic interaction between $\mathrm{H}$ atoms. The $\mathrm{H}-\mathrm{H}$ contribution to the PES is
TABLE I. Parameters used for the mode hydrogen-bonded linear chain. All quantities are expressed in a.u.

\begin{tabular}{cccccccc}
\hline \hline$D$ & $b$ & $r_{e}$ & $k_{\mathrm{F}-\mathrm{F}}$ & $k_{\mathrm{H}-\mathrm{H}}$ & $D_{l}$ & $b_{l}$ & $a_{l}$ \\
\hline 0.0171 & 4.1276 & 1.7763 & 0.02351 & 0.06413 & 0.80848 & 0.3 & 4.4 \\
\hline & $\mathcal{E}_{\mathrm{H}-\mathrm{H}}=$ & $\sum_{i=1}^{N-1} k_{\mathrm{H}-\mathrm{H}}\left(u_{i+1}-u_{i}\right)^{2}+k_{\mathrm{H}-\mathrm{H}}\left(u_{1}-u_{N}\right)^{2}$, &
\end{tabular}

where the last term takes care of PBC. This potential encourages adjacent $\mathrm{H}$ atoms to move the same amount in the same direction, thus promoting the ice rules. The larger the constant $k_{\mathrm{H}-\mathrm{H}}$, the more effectively these are respected. A similar term is included for the $\mathrm{F}$ atoms,

$$
\mathcal{E}_{\mathrm{F}-\mathrm{F}}=\sum_{i=1}^{N-1} k_{\mathrm{F}-\mathrm{F}}\left(\rho_{i+1}-\rho_{i}\right)^{2}+k_{\mathrm{F}-\mathrm{F}}\left(\rho_{1}-\rho_{N}\right)^{2}
$$

with the corresponding PBC. This potential favors the F atoms to move in the same direction, so that two $\mathrm{F}$ atoms cannot approach the same $\mathrm{H}$ at once. This model has been taken from a work by Yanovitskii et al., ${ }^{16}$ who studied the phase diagram in the self-consistent harmonic approximation for a $\mathrm{O}-\mathrm{H} \cdots \mathrm{O}$ linear chain. The only difference with respect to Yanovitskii's model is the value of the harmonic constant $k_{\mathrm{H}-\mathrm{H}}(0.06413$ instead of 0.01413 a.u.). This modification was necessary because the original value did not enforce the ice rules strongly enough, thus leading to a potential with quite unrealistic features such as unstable acoustic phonon branches, as shown in a preliminary version of this work. ${ }^{5}$

This model assumes that the $\mathrm{H}-\mathrm{H}$ and the F-F interactions are independent of the lattice parameter $a$. A mild dependence on the lattice constant enters through the H-F interaction, but it is quite unrealistic. In order to render quantum effects more realistic we have introduced an additional Morse-type potential,

$$
\mathcal{E}_{a}=\sum_{i=1}^{N} D_{l}\left(1-e^{-b_{l}\left(a-a_{l}\right)}\right)^{2} .
$$

The parameters used in the present work were inspired in the calculations presented in Sec. III C and are summarized in Table I. With this choice of parameters, the classical lattice constant is $a_{\mathrm{cl}}=4.368$ bohr. In Fig. 4 we show a schematic picture of the interactions involved in this model.

Figure 5 shows the equilibrium lattice parameter as a function of the inverse number of cells. The behavior of the QHA with the number of cells is similar to the monatomic

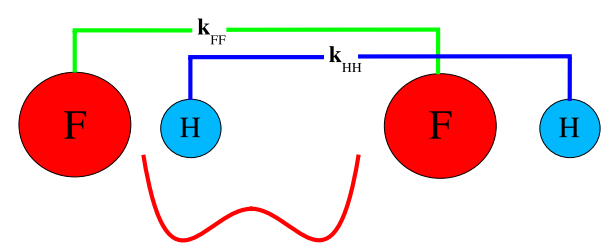

FIG. 4. (Color online) Schematic view of the interactions in a model for a hydrogen-bonded chain. 


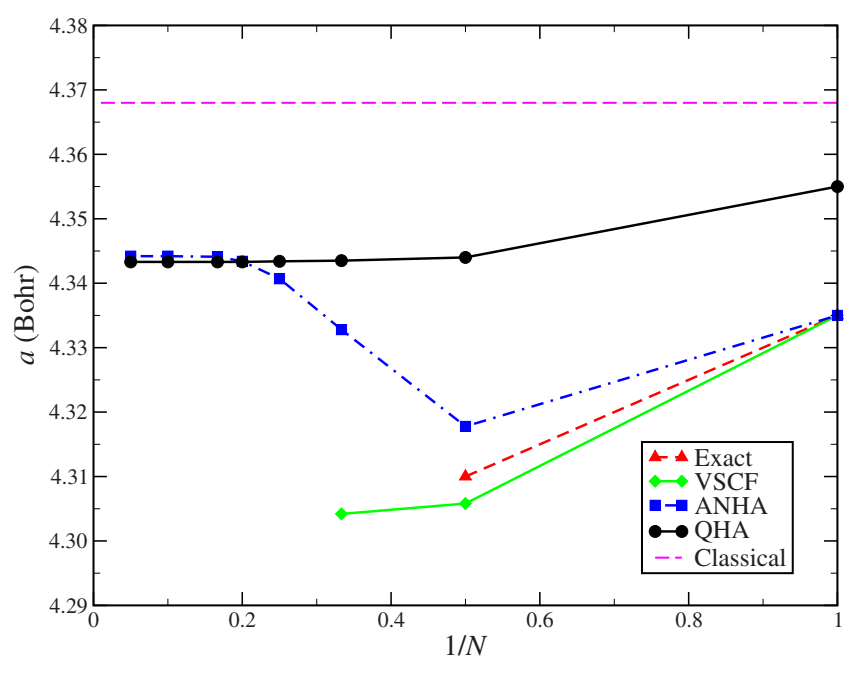

FIG. 5. (Color online) Quantum-corrected lattice constant for a model H-bonded chain as a function of the inverse number of cells. Contrary to covalently bonded systems, the quantum-mechanical effect is to decrease $a$ from its classical value (horizontal longdashed line on top). The green (light gray solid) line with diamonds represents the VSCF results, while the red (short-dashed) line with triangles is the exact result. The blue (dotted-dashed) line with squares is the ANHA, and the black (solid) line with circles is the QHA.

chain (black circles and solid line). Notice that here each cell contains two atoms, so that a well-converged value of $a_{\mathrm{QHA}}$ requires at least four cells, i.e., eight atoms. However, three cells are already quite well converged, and two cells produce a very reasonable value. Therefore, since this is an easily affordable size, in this paper we present results mostly obtained with two cells.

Notice that now $a_{\mathrm{QHA}}$ decreases with increasing $N$. In fact, at variance with the covalently bonded chain, the QHAconverged value is 0.025 bohr smaller than the classical value. This is a characteristic feature of H-bonded systems, where the stretching frequency that contributes to the ZPE initially decreases upon compression because the $\mathrm{H}$ bonds weaken. This occurs until the bond becomes symmetric, and only then the frequencies (and the ZPE) start to increase, as will be shown in Sec. III C.

Next, the normal modes determined at the energy minimum were used to compute the ZPE in the anharmonic approximation (ANHA, blue squares, and dotted-dashed line). The converged value of the lattice constant is essentially indistinguishable from $a_{\mathrm{QHA}}$. Its behavior with $N$, however, is nonmonotonic. This can be explained through the fact that, for this linear chain, there is only one vibrational coordinate that exhibits a double-well potential. As the number of cells is increased, the contribution of this double well to the total energy becomes increasingly unimportant.

In order to evaluate the effect of mode coupling anharmonicities, we carried out VSCF calculations by mapping the PES along the normal modes corresponding to the minimumenergy configuration, i.e., with all the $\mathrm{H}$ atoms off center (green diamonds and solid line at the bottom). The exact calculation used the PES mapped along the normal modes corresponding to the saddle-point configuration, i.e., with all the $\mathrm{H}$ atoms in the center of the bonds (red triangles and dashed line). At variance with the VSCF, the choice of vibrational coordinates is irrelevant because, unlike the case of molecular systems, the vibrational subspace in crystals does not depend on the reference geometry. ${ }^{17}$ In the VSCF approximation we went up to a six-atom supercell $(N=3)$, i.e., a five-dimensional VSCF problem. The good agreement of the VSCF with respect to exact results for $N=2$, together with the trend with $N$ exhibited by the VSCF, suggests that a large fraction of the quantum effect is already captured in the two-cell calculation. Nevertheless, this statement must be taken with caution. Calculations on larger supercells are needed to verify this especially in view of the unusual behavior of the anharmonic approximation ANHA.

Another conclusion is that the QHA underestimates the lattice contraction due to quantum effects remaining at about half the required value. The next level of difficulty and computational cost is the ANHA, but its nonmonotonic behavior with system size makes it rather unsafe as a method to include anharmonicity. In particular, the seemingly good results obtained for two cells appear to be fortuitous. The VSCF, however, appears to be quite a good and robust method that reproduces exact results to an excellent accuracy. This indicates that the correlation between modes is a relatively minor effect, so that a mean-field approximation, where each mode feels the other modes on average, is sufficient for this class of problems.

\section{First-principles hydrogen-bonded chain}

As a realistic application we studied an F-H hydrogenbonded chain by means of first-principles calculations. Actual F-H chains develop a zigzag structure. ${ }^{18}$ However, since our purpose here is not to solve the problem of F-H crystals, but to understand isotope effects when a realistic PES is used, we disregard this geometrical aspect and consider straight chains and the motion of the atoms only along the axis of the chain. First-principles calculations have been carried out using a combination of codes. For phonon calculations we used the pseudopotential plane-wave code Quantum-ESPRESSO. ${ }^{19}$ The energy curves and lattice constants were calculated with SIESTA, ${ }^{20}$ which also uses pseudopotentials but the wave functions are expanded in a localized basis set of pseudoatomic orbitals. All calculations were carried out within the Perdew-Burke-Ernzerhof (PBE) generalized gradient approximation (GGA) (Ref. 21) to densityfunctional theory (DFT).

In Fig. 6 (upper panel) we present the phonon-dispersion relations along the direction of the chain, calculated in the symmetric configuration with the $\mathrm{H}$ atoms in their centered position, from a compressed lattice constant of 4 bohr up to an expanded value of 5.2 bohr. For small $a$ there is no double well. The $\mathrm{H}$ atoms are stable in their centered positions and the optical phonon branch for this reference configuration is stable. At $a \approx 4.22$ bohr the double well emerges and the $\mathrm{H}$ atoms prefer an off-centered configuration. If we insist on calculating the phonon dispersions for the centered configuration, the optical branch develops an instability at zone center. In fact, there is a whole portion of the optical branch that 

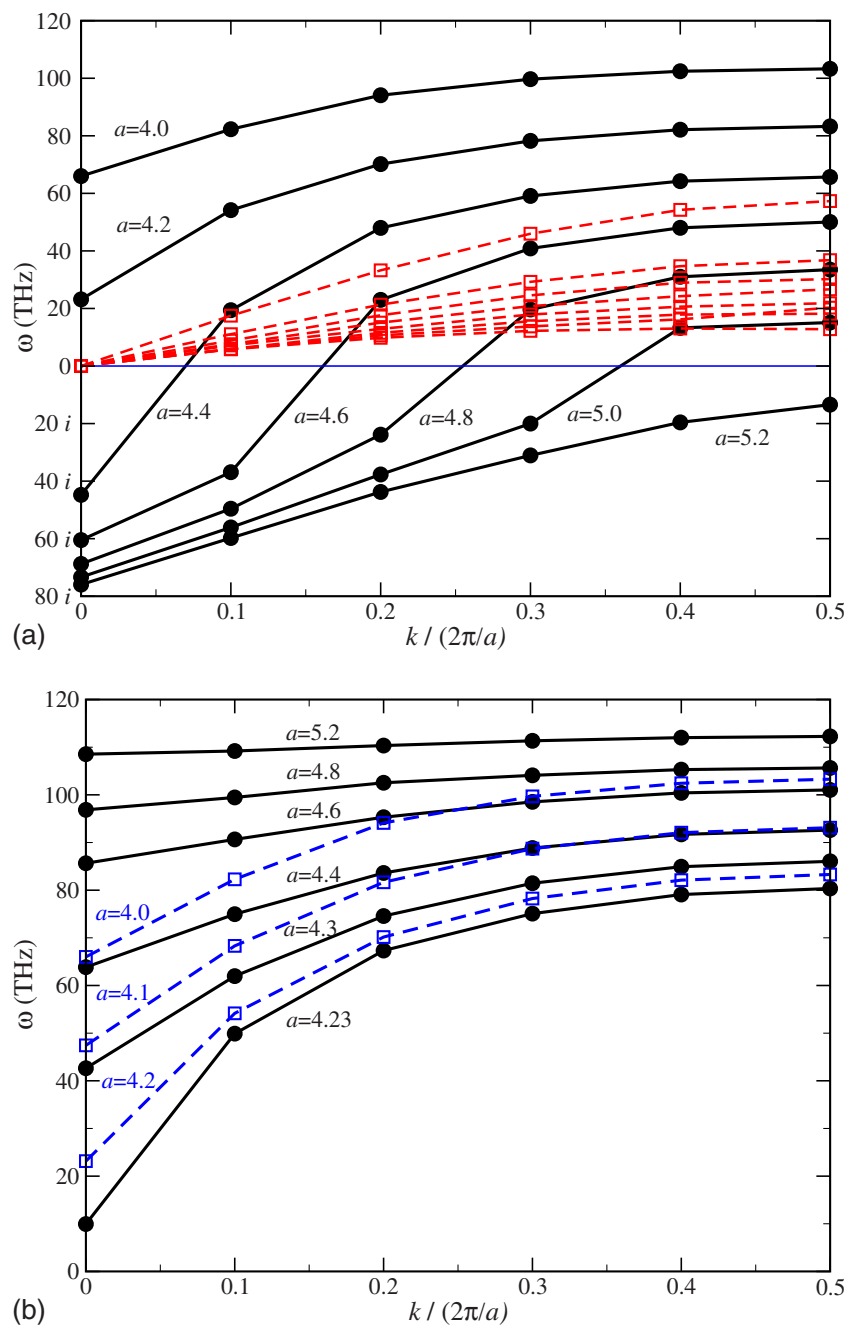

FIG. 6. (Color online) First-principles phonon dispersions for a linear F-H chain at various lattice parameters. Upper panel: $\mathrm{H}$ atoms in centered positions. Red (dashed) lines with open squares represent acoustic branches, while black solid lines with filled circles correspond to optical branches. Lower panel: $\mathrm{H}$ atoms in their stable position. Blue (dashed) lines with open squares correspond to stable centered $\mathrm{H}$ atoms, while black solid lines with filled circles indicate stable off-centered $\mathrm{H}$-atoms. Acoustic branches are omitted for clarity. Values of lattice constants in bohr are indicated in the figure.

is unstable. The extent of the instability region increases with increasing lattice constant to the point of making the optical mode unstable throughout the whole Brillouin zone when the chain is stretched to 5.2 bohr.

Further insight is obtained by calculating the phonondispersion relations for the stable configuration. These are presented in the lower panel of Fig. 6, where it can be observed that the zone-center optical phonon becomes soft at $a \approx 4.22 \mathrm{bohr}$, and after the $\mathrm{H}$ atoms have centered (blue lines and open squares) its frequency starts rising again quite steeply. An interesting observation is that the curvature of the optical phonon branch is the opposite of the usual picture where the frequency decreases from zone center toward zone boundary. This is a consequence of the type of bonding and common to hydrogen-bonded systems. In the zone-center op-
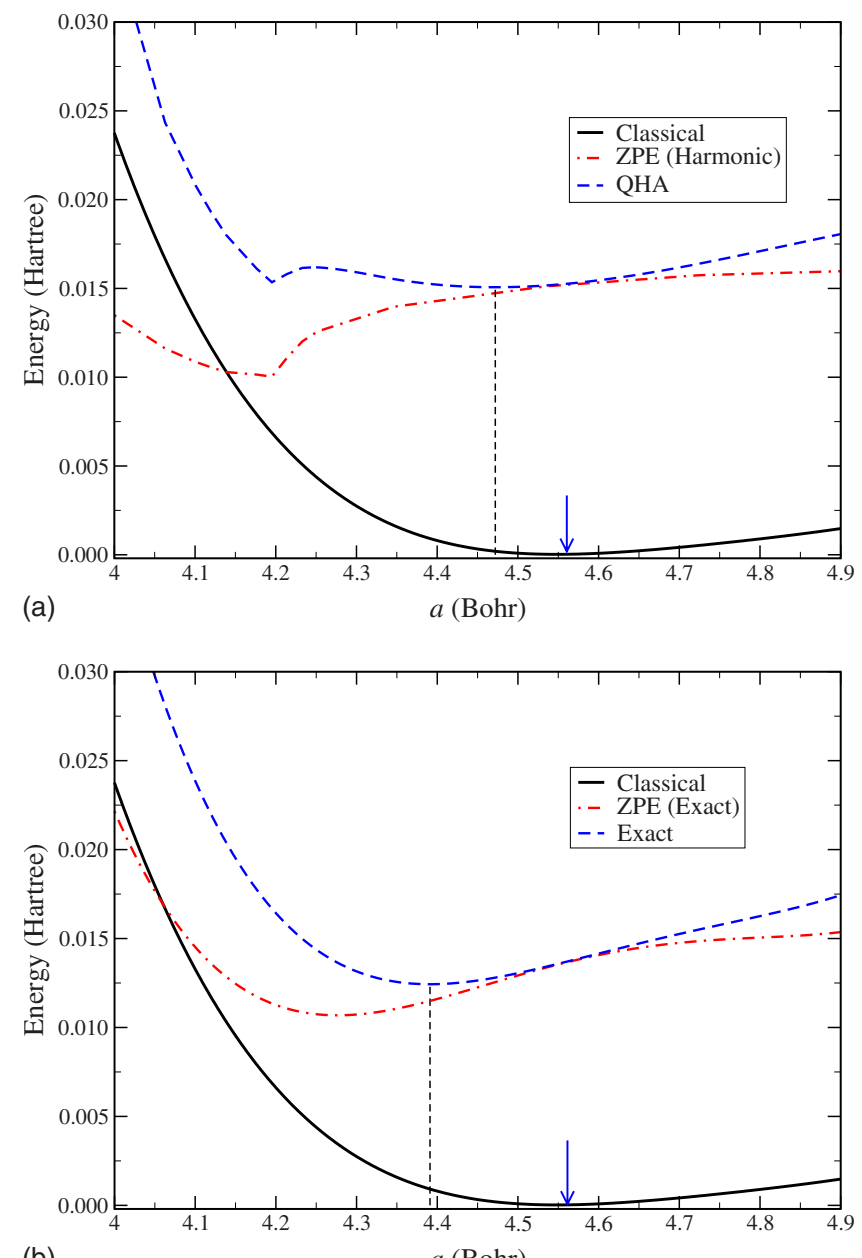

(b)

$a(\mathrm{Bohr})$

FIG. 7. (Color online) Upper panel: harmonic ZPE (red dotteddashed line) and QHA-corrected energy curve (blue dashed line). Lower panel: exact ZPE and quantum-corrected energy as functions of lattice parameter. Color and line coding as in upper panel. The classical energy curve (black solid line) is shown in both panels for comparison. Note the large difference between QHA and exact equilibrium lattice constant (vertical dashed lines). Arrows indicate the classical lattice constant.

tical phonon all unit cells move in phase, with one $\mathrm{H}$ atom approaching the $\mathrm{F}$ atom while the other moves away. At zone boundary, consecutive unit cells move out of phase, so that half of the $\mathrm{F}$ atoms are approached by two $\mathrm{H}$ atoms rather than one. This is in clear violation of the ice rules, which penalize this type of motion with an increased energy, thus explaining why the optical mode is harder at zone boundary.

The harmonic frequencies can now be used to analyze the behavior of the QHA as a function of the lattice constant. In Fig. 7 (upper panel) we show the ZPE (red dotted-dashed line), the classical PES (black solid line), and the quantumcorrected PES (blue dashed line) as functions of the lattice parameter for $N=2$ (four atoms). It is interesting to analyze the behavior of the QHA when the system is highly compressed. It is apparent that the ZPE exhibits a nonanalytic behavior (a cusp) at $a \approx 4.2 \mathrm{bohr}$, where the $\mathrm{H}$ atoms center and the type of bonding changes from hydrogen bonding to covalent. As a consequence, the QHA curve exhibits two 
TABLE II. Equilibrium lattice parameters (in bohr) at various levels of approximation.

\begin{tabular}{cccccc}
\hline \hline No. cells & Classical & QHA & ANHA & VSCF & Exact \\
\hline 1 & 4.560 & 4.195 & 4.381 & 4.381 & 4.381 \\
2 & 4.560 & 4.462 & 4.387 & 4.390 & 4.392 \\
\hline \hline
\end{tabular}

minima; one of which is spurious. Since this characteristic is associated to the stretching mode, and the remaining modes are not severely affected by compression, similar behavior is observed for a larger number of cells. This feature represents a physically incorrect picture and thus a clear limitation of the QHA in the description of hydrogen-bonded systems at high pressures. ${ }^{18}$ In fact, this nonanalytic behavior completely disappears when the problem is solved exactly (lower panel). Notice the substantial difference ( $0.07 \mathrm{bohr}$ ) between the QHA and exact lattice constants (dashed vertical lines).

Similarly to the model presented in Sec. III B, we have examined the dependence of quantum nuclear effects on the number of cells and for the various approximations. Results are summarized in Table II.

For a single cell there is only one vibrational coordinate associated to the F-H stretching, which is subjected to a double-well potential. Since there is only one mode, both ANHA and VSCF approximations are equivalent to the exact solution. The lattice constant obtained in the QHA, however, is anomalously small. By inspection it is clear that this value corresponds to the spurious minimum mentioned above, which for one cell appears to be lower in energy than the correct minimum. Figure 8 shows the quantum-corrected energy as a function of lattice parameter for two cells (i.e., two F-H units, amounting to three vibrational modes).

At variance with a single cell, the QHA (red dotteddashed line) provides a better estimate of the effect. Using

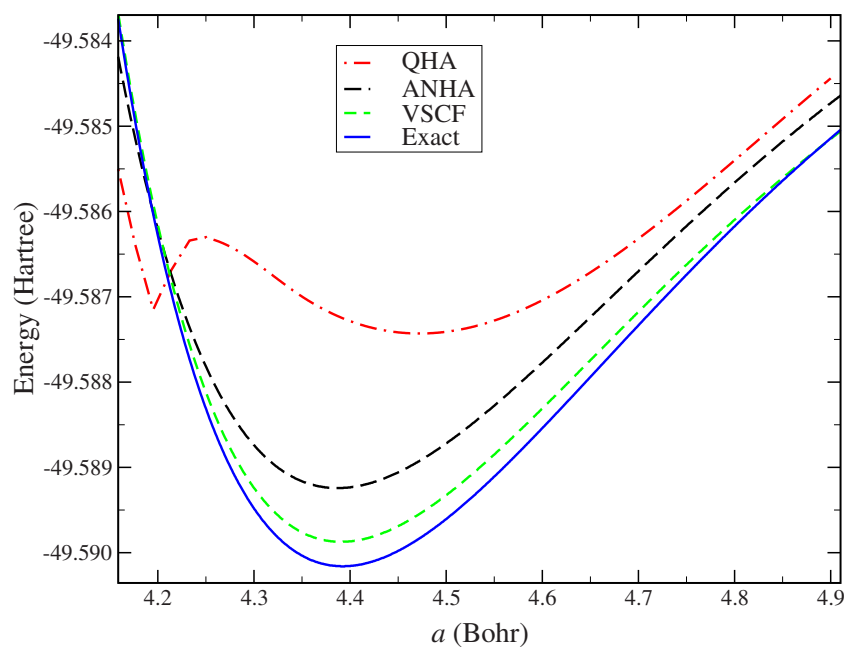

FIG. 8. (Color online) Ground-state energy as a function of lattice parameter for two unit cells at various levels of approximation. The blue (solid) line is for the exact calculation, the green (dashed light gray) line is for the VSCF, the black (long-dashed) line is for the ANHA, and the red (dotted-dashed) line is for the QHA.

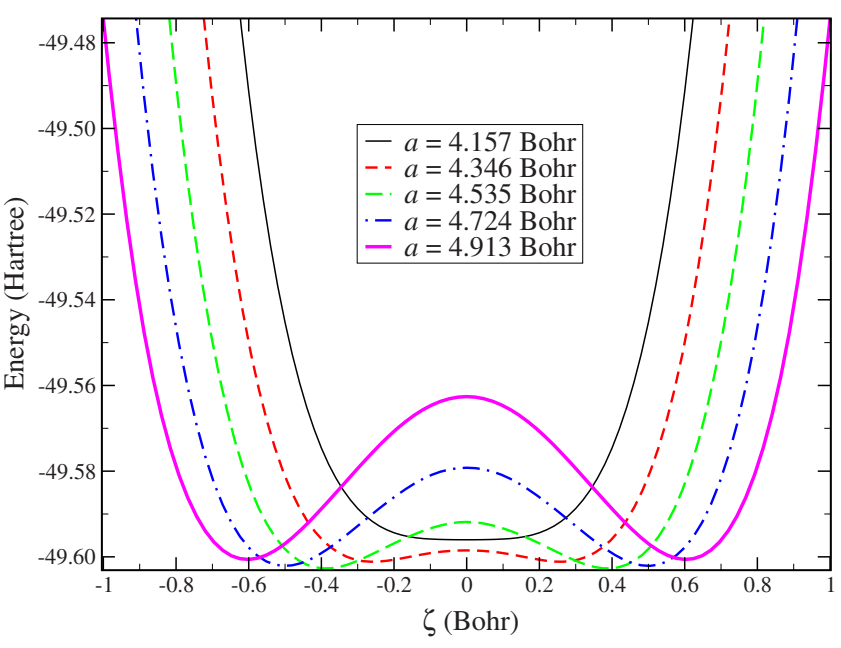

FIG. 9. (Color online) First-principles PES along the zonecenter optical mode for different values of the lattice parameter. The thin black curve represents the most compressed situation at $a$ $=4.157$ bohr. The red curve (dashed) corresponds to $a$ $=4.346 \mathrm{bohr}$, the green (long-dashed light gray) curve to $a$ $=4.535$ bohr (close to the equilibrium value), the blue (dotteddashed) curve to $a=4.724 \mathrm{bohr}$, and the magenta (thick solid, dark gray) curve to an expanded lattice constant of $a=4.913$ bohr. Energies are reported for a supercell containing two unit cells.

the vibrational coordinates obtained in the stable configuration, and already used for the QHA, we introduced intramode anharmonicities through the ANHA (black long-dashed line) and mode coupling anharmonicities via the VSCF approach (green dashed line; light gray in print). The latter is in very good agreement with the exact result (blue solid line) especially for small and large values of the lattice parameter.

In order to assess the quality of the various approximations, we mapped the PES along the zone-center optical mode for different values of the lattice parameter (Fig. 9). For small values of $a$ the $\mathrm{H}$ atoms are centered and subjected to a single-well anharmonic potential (black solid thin line in Fig. 9). Therefore, at high compression the QHA is a good approximation, but closer to the decentering point the potential becomes more anharmonic, thus compromising its quality. This situation is notably improved by introducing intramode anharmonicities in the approximation of noninteracting modes (ANHA). The VSCF approximation reproduces the exact results to an excellent extent, thus indicating that the effect of correlation between modes is very small. A similar trend is observed for large values of the lattice parameter. In this case, the barrier in the double-well potential is so high that the overlap between the two degenerate states (left and right) is very small. Again, the ANHA provides an improved estimate of the energy with respect to the QHA and the VSCF reproduces quite well the exact results, thus indicating that correlation between modes remains negligible. We conclude that in the two limiting cases the ANHA is a rather decent approach and the VSCF reproduces very closely the exact results.

The situation is somewhat different at intermediate values of the lattice parameter, where the barrier is low and the effect of anharmonicity is larger. Here the QHA and ANHA 


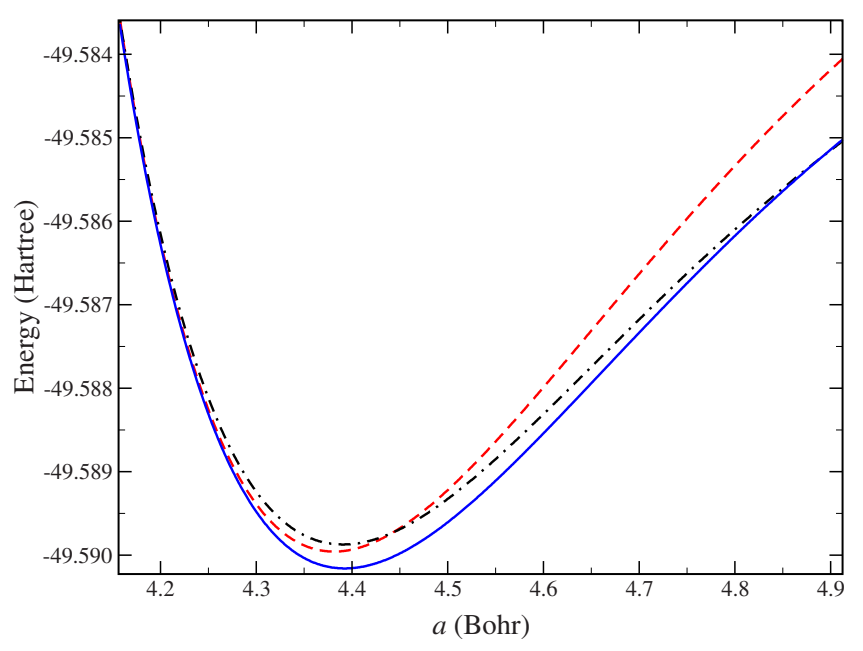

FIG. 10. (Color online) Comparison of the VSCF calculations for two different choices of vibrational coordinates. The red (dashed) line is for the saddle-point modes, while the black (dotteddashed) line is for the modes calculated at the classical equilibrium configuration. The blue solid line represents the exact energy.

are not very satisfactory and have to be improved by introducing mode-coupling anharmonicities at the mean-field level, as in the VSCF scheme. It is also in this region where correlations become more important.

Although the energy curves are shifted, the position of the minimum estimated by the ANHA is very good. According to the results presented in Sec. III B, for the model hydrogenbonded chain, we believe that this may be a fortuitous coincidence for this particular system size. The QHA, however, is evidently not sufficiently accurate.

\section{Choice of vibrational coordinates in the VSCF}

The quality of the VSCF approximation depends on the choice of vibrational coordinates. The more uncorrelated they are, the better the approximation. Normal modes are a good starting point because in the harmonic limit they are completely uncorrelated. However, as the amplitude of the displacements along some soft modes increases (due to quantum delocalization), the normal modes begin to couple. We now analyze the question of which vibrational coordinates are optimal for the VSCF approximation in the case of the four-atom cell. The three modes are the zone-center optical phonon, which exhibits a double well, and the zoneboundary acoustic and optical phonons, which are practically harmonic. In principle, any linear combination of these three modes is possible. Nevertheless, since the modes at different $\mathbf{k}$ vectors do not mix, we fix the double-well mode and optimize the choice of modes in the two-dimensional zoneboundary subspace. ${ }^{22}$

We studied the quality of the VSCF for two different choices: the normal modes calculated at the minimum of the PES and those calculated at the saddle-point configuration, with the $\mathrm{H}$ atoms centered. The VSCF energies are reported in Fig. 10 together with the exact energy. According to Fig. 9, the two minima in the double well get closer and eventually merge as the lattice parameter is reduced. Therefore, for values of $a$, where the double well has disappeared, there is no saddle point and the normal modes at the minimum are an excellent choice for the VSCF. Similarly, at large $a$ the barrier separating the two minima is high, and again the wave function is quite localized around the two minima.

Therefore, the vibrational coordinates calculated at the minimum of the PES are generally better than the saddlepoint modes for solving the VSCF problem. At intermediate values of $a$ none of the two choices is clearly superior to the other. We have tried to optimize the choice of modes within this subspace by minimizing the ground-state VSCF energy, but the energy gain was always of the same order of magnitude of the difference between minimum and saddle-point modes. Therefore, this appears to be the limit of the VSCF. Any further improvement requires the introduction of correlation between modes. The present situation is reminiscent of static correlation cases often encountered in electronic structure calculations, where a single Slater determinant (an uncorrelated electronic configuration) is insufficient to represent the ground state. These require a multideterminantal wave function as in multireference methods such as complete active space self-consistent field (CASSCF). Here, a linear combination of two Hartree products, each one corresponding to one of the two equivalent minima, constitutes an improved wave function that correctly respects the symmetry of the problem. It is well known that static correlation cannot be recovered by perturbative methods based on a single reference. ${ }^{23}$ This is also a problem for truncated configuration interaction (CI) expansions, while full $\mathrm{CI}$ would require a large basis set. This is why, in the field of molecular spectroscopy, various groups have developed multiconfiguration methods such as multi-component time-dependent Hartree $(\mathrm{MCTDH}){ }^{24}$

\section{E. Isotope effects}

Figure 11 shows the effect of modifying the nuclear masses on the lattice parameter for the case of two F-H cells. In order to evaluate isotope effects, both the $\mathrm{H}$ and $\mathrm{F}$ masses were multiplied by a scale factor $s$, so that the mode eigenvectors, and thus the vibrational coordinates, were not modified. We have then solved the three-dimensional Schrödinger equation in the vibrational space for various lattice constants and obtained the quantum corrected energy curves for the true $\mathrm{H}$ and $\mathrm{F}$ masses (red short-dashed line) when these masses are multiplied by 2 (violet dotted line), 5 (green longdashed line), and 10 (blue dotted-dashed line). For comparison we also show the classical energy curve, corresponding to the limit of infinite masses. As expected, the energy increases with decreasing mass, while the lattice constant decreases, as illustrated in the inset. This is the essence of the geometric or Ubbelohde effect in H-bonded systems. ${ }^{25}$ Lighter particles are more delocalized and exhibit a larger probability in the region of the barrier. According to Fig. 9, this favors more compressed bonds, thus translating into an effective attraction between the two neighboring $\mathrm{F}$ atoms (or oxygens in the more common $\mathrm{O}-\mathrm{H} \cdots \mathrm{O}$ hydrogen bonds). This attraction is reflected in an enhanced cohesion, and thus in a smaller lattice constant,${ }^{26}$ which is a common feature of 


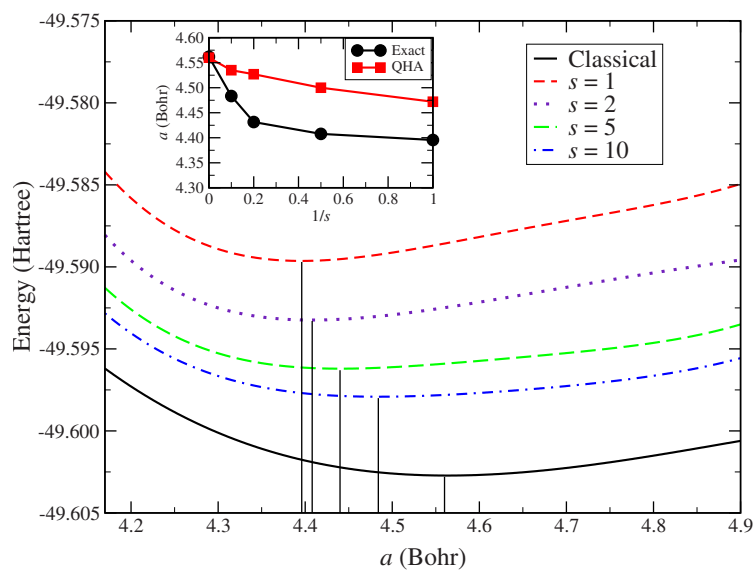

FIG. 11. (Color online) Energy vs lattice constant for various mass-scaling factors. The black (solid) line is the classical result, blue (dotted-dashed) line is for $s=10$, green (long-dashed light gray) line for $s=5$, violet (dotted) line for $s=2$, and red (shortdashed dark gray) line for $s=1$. Vertical lines indicate the equilibrium lattice constants, which are then reported in the inset as a function of $1 / s$. The H-F chain PES has been calculated at the DFT-PBE level, using the SIESTA code. Actual H-F chains assume a zigzag form, but here we considered linear chains.

a large family of H-bonded crystals. ${ }^{27}$ To illustrate this concept of enhanced delocalization, we show in Fig. 12 a twodimensional cut of the wave function for the true masses and those multiplied by 5 for a lattice constant $a=4.44$ bohr.

We can now use these wave functions to calculate the quantum expectation value of the F-H distance as a function of the mass of the particles. This is a very important quantity because it is what obtained in neutron and x-ray diffraction experiments. This distance is usually quite different from that obtained classically for the same lattice parameter, which corresponds to the minimum of the double-well PES. In fact, this is probably the source of many inconsistencies between experimental structures and those determined via firstprinciples calculations. ${ }^{26}$ In Table III we report the exact F-H distance as a function of the mass-scaling factor for a lattice constant of 4.44 bohr. As expected, the F-H distance decreases when the masses become heavier. The classical F-H distance increases by a substantial $10 \%$ when the true $\mathrm{H}$ and $\mathrm{F}$ masses are used. In contrast, at fixed lattice constant the

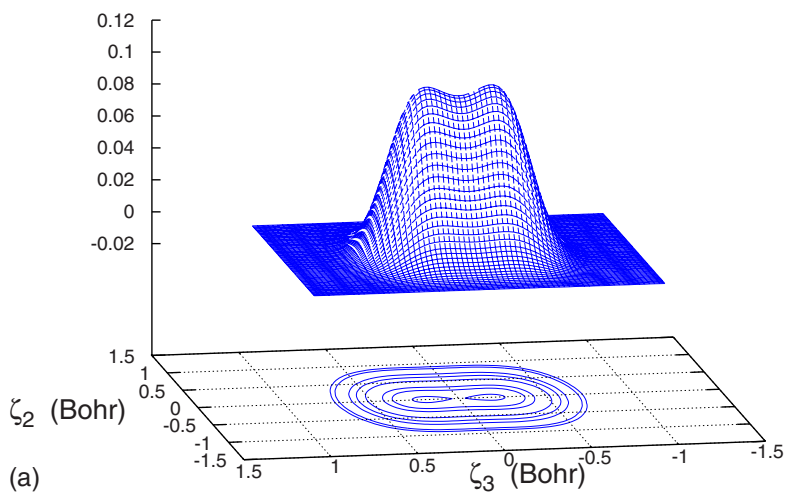

TABLE III. Equilibrium F-H distance (in bohr) as a function of the mass-scaling factor $s$ for a lattice constant of $4.44 \mathrm{bohr}$

\begin{tabular}{cccccc}
\hline \hline$s$ & 1 & 2 & 5 & 10 & $\infty$ \\
\hline$d_{\mathrm{F}-\mathrm{H}}$ & 2.08 & 2.06 & 2.02 & 1.99 & 1.85 \\
\hline \hline
\end{tabular}

QHA values are insensitive to $s$. This is because quantum and classical harmonic oscillators have the same equilibrium position, and thus the quantum harmonic approximation does not modify the internal geometry. More generally, the F-H distance in the QHA corresponds to the classical value obtained at the $s$-dependent equilibrium lattice constant, which is reported in the inset of Fig. 11 (red symbols). Therefore, the influence of mass scaling is limited to the volume effect, which is significantly smaller than the full quantum effect. First, the dependence of the QHA lattice constant with $s$ is milder than the exact one. Second, as shown in Fig. 9, variations of this magnitude lead to small changes in the location of the minima of the double well. As a consequence, the F-H distance is severely underestimated in the QHA, thus precluding its use in the description of the internal geometry of $\mathrm{H}$-bonded systems.

In the present case, the isotope effect upon doubling of all the masses, which is closely related to deuteration, does not entail a significant change in the F-H distance. In a first instance one could think that this is because it was calculated at fixed lattice constant (see Table III). In effect, this would be consistent with theoretical calculations ${ }^{26}$ and experiments under pressure. ${ }^{28}$ When sufficient pressure is applied to deuterated compounds to reproduce the lattice parameters of its protonated analog, the internal geometries turn out to be quite similar. Within this context, one of us has shown that most of the isotope effect arises from a self-consistent interplay between wave-function localization, internal geometry, and lattice parameters. ${ }^{29}$ Therefore, to assess the full extent of the isotope effect, one would have to compare the internal geometries at the corresponding equilibrium lattice constants. In the present case we obtained F-H distances of 2.12 and $2.10 \mathrm{bohr}$, respectively. Interestingly, the isotope effect remains as small as before. This is not inconsistent with experimental data, though. In fact, the magnitude of the isotope effect on interatomic distances in hydrogen-bonded systems

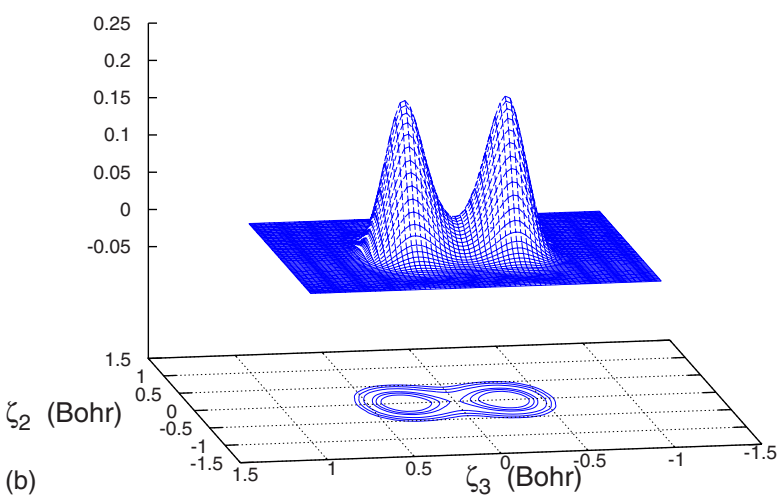

FIG. 12. (Color online) Two-dimensional cut of the ground-state wave function in the subspace of the two optical modes $\zeta_{2}$ and $\zeta_{3}$. The upper panel is for the true masses and the lower panel is for particles five times heavier. Notice the enhanced localization of the latter. 
ranges from almost insignificant values for some systems to large differences as in $\mathrm{KH}_{2} \mathrm{PO}_{4}$, where the $\mathrm{O}-\mathrm{O}$ distance of $2.45 \AA$ rises to $2.52 \AA$ in its deuterated analog $\mathrm{KD}_{2} \mathrm{PO}_{4} \cdot{ }^{28}$ This depends on several factors, mainly the size of the coupling between internal coordinates and strain and the shape of the double well (barrier height and distance between minima) in the region around the equilibrium lattice constant.

\section{CONCLUSIONS AND OUTLOOK}

We have analyzed the influence of quantum nuclear effects on the structural properties of solids. To this end we have implemented a simple methodology of mapping the multidimensional PES in the space of normal modes and then solving the resulting vibrational Schrödinger equation. This has been done exactly when possible and also using a number of computationally tractable approximations that range from the vibrational self-consistent field (VSCF) method and its variants down to the quasiharmonic approximation (QHA).

We have studied the behavior of energies and lattice constants in an anharmonic monatomic chain as a function of the size of the supercell and showed that results for four cells are probably already quite close to convergence. Here, the QHA is an excellent approximation unless the masses become exceedingly small, thus justifying the customary approach followed in solid-state physics. We have then analyzed the behavior of a model hydrogen-bonded chain and observed that the coupling between modes can be important. Finally, we considered a realistic $\mathrm{H}$-bonded chain where the PES was determined from first-principles calculations. Here we obtained the quantum-corrected lattice constant and internal structural parameters (distances) and showed how isotope effects arise in these systems. We have also analyzed the various approximations and showed that the QHA is insufficient, while the independent-mode anharmonic approximation (ANHA) appears to introduce an important improvement at the energetic and structural levels. Nevertheless, results obtained for the model hydrogen-bonded chain suggest that this may be accidental and due to size effects. VSCF results are very close to exact ones, thus suggesting that correlation between modes is a minor effect. Optimizing the vibrational coordinates in the VSCF method does not constitute a significant advantage; further improvement should arise from correlated methods such as vibrational CI or MCTDH. In any case, the VSCF approach using the modes calculated at the classical equilibrium geometry appears to be a very good approximation.

Although technically the extension to higher dimensionalities is straightforward, there are some features that are not present in one-dimensional systems. For example, the tunneling mode can be strongly coupled to other modes, ${ }^{6}$ so that, for large amplitude of motion, correlation between them may become important. In this case we enter the realm of multidimensional tunneling where, if we insist on describing the proton motion in terms of a single effective tunneling coordinate, the latter tends to be curvilinear. Therefore, it is quite likely to have to adopt schemes that combine the exact solu- tion for one or a few subspaces of strongly coupled modes, with a VSCF representation or a lower-level scheme such as the QHA for the remaining modes. ${ }^{30}$ It remains the problem of identifying what are those subspaces though.

There are many aspects of this approach that can be improved especially for what concerns simplified methods that can be used for larger systems and higher dimensionality. Within the VSCF scheme, the limiting factor is the mapping of the PES and the multidimensional integration. The latter can be efficiently dealt with by factorizing the PES into a sum of products of modes. ${ }^{8}$ This allows for the computation of VSCF potentials as products of inexpensive onedimensional integrals. The problem of fitting the PES remains open, although there are general strategies for PES fitting based on interpolation methods ${ }^{31}$ or using principles of multivariate analysis such as the high-dimensional model representation of Rabitz and Aliş. ${ }^{32}$ An improvement to the ANHA that approximately recovers the interaction between modes at the mean-field level consists of using the quantum expectation value of the geometry as a reference rather than the classical one. This can also be seen as a simplification of the VSCF approximation where the vibrational wave functions in the integrals are replaced by completely localized delta functions. This is a good approximation unless one or more approximated wave functions exhibit two peaks corresponding to a double-well potential.

Another subtle issue in hydrogen-bonded systems is that, although DFT-GGA approaches reproduce structural properties quite well, proton transfer barriers appear to be exceedingly small in some cases even disappearing altogether. ${ }^{33}$ Therefore, other electronic structure methods such as hybrid Hartree-Fock DFT (Ref. 34) or correlated quantum chemical methods have to be explored. ${ }^{35}$

A straightforward extension of this methodology is the calculation of vibrational excitations. Once the PES is known, excited states can be easily calculated and used to compute thermodynamic quantities beyond the quasiharmonic approximation. General VSCF algorithms useful to tackle mild anharmonicities in molecular systems have been implemented many years ago ${ }^{13}$ and more recently in conjunction with an $a b$ initio description of the PES. ${ }^{36}$ In fact, a VSCF option is available in some electronic structure codes such as GAMESS. ${ }^{37}$ The main use of this capability is at the spectroscopic and thermochemical rather than structural level. An extension of this methodology to crystalline systems is straightforward and perfectly viable, although to the best of our knowledge this has not been implemented so far. Nevertheless, the treatment of highly anharmonic systems such as those involving double wells remains a challenge. We expect the present paper to be a relevant contribution in this direction.

\section{ACKNOWLEDGMENTS}

We thank David Hughes and Alfredo Caro for important contributions in the initial stages of this project. We also thank Pietro Ballone, Mario Del Popolo, and Ricardo Migoni for helpful discussions. 
*j.kohanoff@qub.ac.uk

${ }^{1}$ G. Liebfried and W. Ludwig, Solid State Physics (Academic, New York, 1961), Vol. 12, p. 276.

${ }^{2}$ R. A. Cowley, Adv. Phys. 12, 421 (1963).

${ }^{3}$ L. N. Kantorovich, Phys. Rev. B 51, 3520 (1995).

${ }^{4}$ C. Kittel, Introduction to Solid State Physics (Wiley, New York, 1986).

${ }^{5}$ I. Scivetti, D. Hughes, N. I. Gidopoulos, A. Caro, and J. Kohanoff, Computational Methods in Science and Engineering Vol. 1 - Theory and Computation: Old Problems and New Challenges, AIP Conf. Proc. No. 963 (AIP, New York, 2007), p. 212.

${ }^{6}$ G. Colizzi, Ph.D. thesis, Queen's University Belfast, 2005.

${ }^{7}$ S. Baroni, S. de Gironcoli, A. Dal Corso, and P. Giannozzi, Rev. Mod. Phys. 73, 515 (2001).

${ }^{8}$ A. Jäckle and H.-D. Meyer, J. Chem. Phys 104, 7974 (1996); 109, 3772 (1998).

${ }^{9}$ M. A. Suhm and R. O. Watts, Phys. Rep. 204, 293 (1991).

${ }^{10}$ D. Baye and P.-H. Heenen, J. Phys. A 19, 2041 (1986); D. Baye, Phys. Status Solidi B 243, 1095 (2006)

${ }^{11}$ K. Varga, Z. Zhang, and S. T. Pantelides, Phys. Rev. Lett. 93, 176403 (2004).

${ }^{12}$ M. A. Ratner and R. B. Gerber, J. Phys. Chem. 90, 20 (1986).

${ }^{13}$ A. Roitberg, R. B. Gerber, R. Elber, and M. A. Ratner, Science 268, 1319 (1995)

${ }^{14}$ T. C. Thompson and D. G. Truhlar, J. Chem. Phys. 77, 3031 (1982).

${ }^{15}$ A. Hidalgo, J. Zúñiga, J. M. Francés, A. Bastida, and A. Requena, Int. J. Quantum Chem. 40, 685 (1991).

${ }^{16}$ O. Yanovitskii, G. Vlastou-Tsinganos, and N. Flytzanis, Phys. Rev. B 48, 12645 (1993).

${ }^{17}$ I. Scivetti, Ph.D. thesis, Queen's University Belfast, 2008.

${ }^{18}$ R. W. Jansen, R. Bertoncini, D. A. Pinnick, A. I. Katz, R. C. Hanson, O. F. Sankey, and M. O'Keeffe, Phys. Rev. B 35, 9830 (1987).

${ }^{19}$ Quantum-ESPRESSO is a community project for high-quality quantum-simulation software, based on density-functional theory, and coordinated by Paolo Giannozzi. See http:// www.quantum-espresso.org
${ }^{20}$ J. M. Soler, E. Artacho, J. Gale, A. García, J. Junquera, P. Ordejón, and D. Sánchez-Portal, J. Phys.: Condens. Matter 14, 2745 (2002).

${ }^{21}$ J. P. Perdew, K. Burke, and M. Ernzerhof, Phys. Rev. Lett. 77, 3865 (1996).

${ }^{22}$ Notice that for larger supercells there will be coupling between zone-center optical modes. In the case of higher dimensionality, this coupling may introduce a curvature in the double-well coordinate that has to be treated carefully.

${ }^{23}$ L. Norris, M. A. Ratner, A. E. Roitberg, and R. B. Gerber, J. Chem. Phys. 105, 11261 (1996).

${ }^{24}$ H.-D. Meyer, U. Manthe, and L. S. Cederbaum, Chem. Phys. Lett. 165, 73 (1990).

${ }^{25}$ J. M. Robertson and A. R. Ubbelohde, Proc. R. Soc. London, Ser. A 170, 222 (1939).

${ }^{26}$ S. Koval, J. Kohanoff, J. Lasave, G. Colizzi, and R. L. Migoni, Phys. Rev. B 71, 184102 (2005).

${ }^{27}$ R. Blinc and B. Zeks, in Soft Modes in Ferroelectrics and Antiferroelectrics, edited by E. P. Wohlfarth (North-Holland, Amsterdam, 1974)

${ }^{28}$ R. J. Nelmes, Z. Tun, and W. F. Kuhs, Ferroelectrics 71, 125 (1987).

${ }^{29}$ S. Koval, J. Kohanoff, R. L. Migoni, and E. Tosatti, Phys. Rev. Lett. 89, 187602 (2002).

${ }^{30}$ O. Vendrell, V. Gatti, V. Lauvergnat, and H.-D. Meyer, J. Chem. Phys. 127, 184302 (2007).

${ }^{31}$ T.-S. Ho and H. Rabitz, J. Chem. Phys. 104, 2584 (1996).

${ }^{32}$ H. Rabitz and Ö. F. Aliş, J. Math. Chem. 25, 197 (1999).

${ }^{33}$ S. Sadhukhan, D. Muñoz, C. Adamo, and G. E. Scuseria, Chem. Phys. Lett. 306, 83 (1999).

${ }^{34}$ B. J. Lynch and D. G. Truhlar, J. Phys. Chem. A 105, 2936 (2001).

${ }^{35}$ L. Maschio, D. Usvyat, F. R. Manby, S. Casassa, C. Pisani, and M. Schütz, Phys. Rev. B 76, 075101 (2007).

${ }^{36}$ G. M. Chaban, J. O. Jung, and R. B. Gerber, J. Chem. Phys. 111, 1823 (1999).

${ }^{37} \mathrm{http} / / /$ www.msg.chem.iastate.edu/GAMESS/GAMESS.html 